\title{
Identificação de Fontes de Resistência em Cultivares de Milho à Spodoptera frugiperda (J. E. Smith, 1797) em Casa- de-Vegetação
}

\author{
Deise Maria P. da Silva*, José V. de Oliveira, José Nildo Tabosa, Reginaldo Barros, Elton \\ O. dos Santos e Simone S. Azevedo. \\ EPEAL/ IPA / Laboratório de Bioinsumos, cx. Postal 1022, CEP. 50761-000 - Recife - PE; Universidade Federal \\ Rural de Pernambuco / Departamento de Agronomia - Fitossanidade, CEP. 52.171-900 - Recife - PE
}

\begin{abstract}
Two experiments were conducted in a greenhouse, with the objective of identifying sources of resistance in 15 corn cultivars to Spodoptera frugiperda. The following cultivars were utilized CMS-35 , CMS-36, Dina-70, Cargill-525, Pioneer-3210, Pioneer-6875, BR-201, BR-451, AG-106, AG-510, CMS-22, Contimax-322, BR-201, Jatinã C 3 Anão, Cargill-511 e BR-106. When the plants had 8 to 10 leaves they were infested with 30 newly ecloded larvae per plant. Damage evaluation was made 10 to 14 days after infestation, in both experiments, using a visual note scale from 0 to 9. There were no statistical differences among cultivars for the parameters evaluated damage, weight, length and survival larvae. Nevertheless a significant linear correlation was found between weigth $x$ larvae length $(n=0,7776$, $P=0,01)$, demonstrating that these parameters can be utilized in the study of corn resistence to $\mathrm{S}$. frugiperda.
\end{abstract}

Key words: Resistance, Zea mays, Foliar Damage, Fall armyworm, Larvae weight

\section{INTRODUÇÃO}

Spodoptera frugiperda (J. E. Smith, 1797) ocorre nos diferentes estádios de desenvolvimento do milho, sendo considerada a sua principal pragachave (Cruz, 1993). As perdas dependem do nível de infestação e do estádio fenológico da cultura, sendo mais expressivas quando as plantas apresentam de 8 a 10 folhas (Cruz \& Turpin, 1982).

O uso de cultivares resistentes constitui uma das táticas mais importantes no Manejo Integrado de S. frugiperda em milho, sendo compatível com outros métodos de controle, além de trazer vantagens econômicas, ecológicas e toxicológicas para os produtores e manutenção do equilíbrio para o agroecossistema.

O conhecimento das técnicas de criação massal, infestação artificial e escala visual de notas (Mihm, 1983, 1984; Wiseman, 1985) bem como, a avaliação da duração das fases larvais, sobrevivência de lagartas, peso pupal (Williams et al., 1978) e mecanismos de resistência, constituem requisitos básicos para o desenvolvimento de programas de melhoramento de milho visando resistência a esta praga.

Nos últimos anos, pesquisas a nível nacional e internacional sobre resistência de milho a $S$. frugiperda foram bastante intensificadas, utilizando-se os mecanismos de não preferência (Wiseman et al., 1967; Silva et al., 1969; Widstrom et al., 1972; Wiseman et al., 1981; Wiseman \& Widstrom, 1986; Wilson et al., 1991) e antibiose (NG, Davis \& Williams, 1985; Melo \& Silva, 1987; Wiseman \& Isenhour, 1988; Cruz \& Alvarenga, 1990; Yang et al., 1991; Viana \& Potenza, 1991; Videla et al., 1992).

Objetivou-se com este trabalho, identificar cultivares de milho portadoras de fontes de resistência à $S$. frugiperda, visando sua utilização em programas de manejo integrado. 


\section{MATERIAL E MÉTODOS}

Foram conduzidos dois experimentos em casade-vegetação da Área de Fitossanidade do Departamento de Agronomia da Universidade Federal Rural de Pernambuco - UFRPE, utilizando-se as cultivares de milho CMS-35, CMS-36, Dina-70, Cargill-525, Pioneer-3210, Pioneer-6875, BR-201, BR-451, AG-106, AG510, CMS-22, Contimax-322, Jatinã $C_{3}$ Anão, Cargill-511 e BR-106, fornecidas pela Empresa Pernambucana de Pesquisa Agropecuária - IPA. As lagartas de S. frugiperda foram criadas no Laboratório de Biologia de Insetos da UFRPE, em folhas de milho da cultivar Centralmex, com idade variando entre 20 e 40 dias.

$\mathrm{O}$ primeiro experimento foi instalado segundo o delineamento experimental de blocos ao acaso com 15 tratamentos e 6 repetições, no período de julho a agosto de 1993. As temperaturas e umidades relativas médias registradas nesses meses foram $22,1{ }^{\circ} \mathrm{C} ; 79 \%$ e $24,6{ }^{\circ} \mathrm{C} ; 69 \%$, respectivamente. As plantas foram semeadas em vasos de cerâmica de 15 x $16 \mathrm{~cm}$, com capacidade para $3 \mathrm{~kg}$ de solo, sendo cada parcela constituída de duas plantas. Quando estas atingiram o estágio de crescimento de seis a oito folhas, foram infestadas artificialmente com 30 lagartas neonatas da geração $\mathrm{F}_{1}$ Videla et al, (1992) e distribuídas nas quatro primeiras folhas do terço superior das plantas, através do método do pincel (Morril \& Greene, 1974).

Decorridos 14 dias após infestação foram atribuídas notas em cada planta, utilizando-se a escala visual de Davis \& Williams (1989). Em seguida, as plantas foram dissecadas para avaliação das lagartas sobreviventes, peso e comprimento das lagartas.

Os resultados foram submetidos á análise de variância e as médias comparadas pelo teste de
Tukey, ao nível de 1 e $5 \%$ de probabilidade. Os dados de sobrevivência das lagartas foram transformados em Arc sen $\sqrt{100}$. Foram ainda efetuadas análises de correlaçấo linear simples entre as notas conferidas às plantas, sobrevivência, peso e comprimento das lagartas.

O segundo experimento foi conduzido no período de setembro $\left(25{ }^{\circ} \mathrm{C}\right.$ e $60 \%$ UR) a outubro $(26,8$ ${ }^{\circ} \mathrm{C}$ e $57 \%$ UR), utilizando-se o mesmo delineamento experimental e metodologias, porém as avaliações foram efetuadas após 10 dias da infestação artificial das lagartas.

\section{RESULTADOS E DISCUSSÃO}

Os dados referentes aos danos, sobrevivência, peso e comprimento de lagartas de $S$. frugiperda quando avaliados aos 14 dias após a infestação, revelaram que não houve diferenças significativas entre as 15 cultivares de milho testadas $(\mathrm{P}<0,05)$ (Tabela 1). No entanto, observou-se uma maior variação principalmente no peso das lagartas, onde aquelas criadas na cultivar CMS-36 apresentaram o menor peso (90,50 mg) em relação a BR-451 (166,50 mg), fato que pode evidenciar na primeira cultivar, presença de alomônios responsáveis pela resistência do tipo antibiose. Resultados contrários foram obtidos por Wiseman et al. (1966), quando detectaram em condições de casade-vegetação, resistência para os genótipos Faw $\neq 1$ e Texas Exp. Hy 6717 entre 217 materiais avaliados.

Constatou-se apenas uma correlação linear positiva e significativa entre $\mathrm{o}$ peso $\mathrm{e}$ comprimento de lagartas $(\mathrm{r}=0,7810, \mathrm{P}=0,01)$, demonstrando que esses dois parâmetros podem ser utilizados na avaliação da resistência de milho à S. frugiperda (Tabela 2). 
Tabela 1. Médias dos danos (nota), sobrevivência (\%), peso (mg) e comprimento de lagartas (mm) de Spodoptera frugiperda em 15 cultivares de milho, 14 dias após infestação artificial em casa-de-vegetação. Recife, PE, de julho a agosto de 1993.

\begin{tabular}{lcccc}
\hline \multicolumn{1}{c}{ Cultivar } & Dano & Sobrevivência $^{*}$ & Peso & Comprimento \\
\hline CMS-35 & 7,58 & 6,62 & 133,67 & 20,70 \\
CMS-36 & 8,42 & 4,85 & 90,83 & 17,83 \\
Dina-70 & 8,42 & 5,25 & 104,17 & 19,20 \\
Cargill-525 & 8,08 & 3,92 & 121,23 & 20,60 \\
Pioneer 3210 & 8,33 & 4,40 & 108,33 & 20,33 \\
Pioneer 6875 & $8, .42$ & 4,40 & 142,17 & 21,48 \\
BR-201 & 8,42 & 4,40 & 109,67 & 20,28 \\
BR-451 & 8,08 & 4,14 & 166,50 & 22,03 \\
AG-106 & 8,00 & 5,52 & 119,00 & 20,18 \\
AG-510 & 7,75 & 4,40 & 102,33 & 18,78 \\
CMS-22 & 7,83 & 3,85 & 129,33 & 21,55 \\
Contimax-322 & 8,08 & 4,85 & 118,50 & 19,18 \\
JatinãC 3 Anão & 7,67 & 4,23 & 103,50 & 19,57 \\
Cargill 511 & 8,08 & 5,80 & 112,67 & 20,20 \\
BR-106 & 7,92 & 4,41 & 101,83 & 19,65 \\
\hline F & $\mathrm{NS}$ & $\mathrm{NS}$ & $\mathrm{NS}$ & $\mathrm{NS}$ \\
C.V. ( \% ) & 7,57 & 22,80 & 30,66 & 13,23 \\
\hline
\end{tabular}

* Dados transformados em Arc sen $\sqrt{\frac{p}{100}}$

Tabela 2. Coeficientes de correlação linear simples entre os parâmetros utilizados na avaliação da resistência de cultivares de milho à Spodoptera frugiperda, em casa-de-vegetação. Recife, PE, de julho a outubro de 1993

\begin{tabular}{lcccccc}
\hline & \multicolumn{5}{c}{ Parâmetros } \\
\hline Exp. & DPxSL & DPxPL & DPxCL & SLxCL & SLxCL & PLxCL \\
\cline { 2 - 7 } 14 dias & $0,0584_{\mathrm{NS}}$ & $0,1649_{\mathrm{NS}}$ & $0,0991_{\mathrm{NS}}$ & $-0,0731_{\mathrm{NS}}$ & $-0,0245_{\mathrm{NS}}$ & $0,7810^{* *}$ \\
10 dias & $-0,3738^{* *}$ & $0,4976^{* *}$ & $0,4492 * *$ & $-0,3977 * *$ & $-0,2783^{* *}$ & $0,7776^{* *}$ \\
\hline
\end{tabular}

DP = Dano na planta (nota)

$\mathrm{SL}=$ sobrevivência da lagarta

$\mathrm{PL}=$ Peso da lagarta

$\mathrm{CL}=$ Comprimento da lagarta

** = Significativo ao nível de $1 \%$ de probabilidade

Para o segundo experimento também não foram encontradas diferenças significativas entre os tratamentos, no entanto, os danos mostraram-se menores e mais variáveis, em relação às observações efetuadas aos 14 dias (Tabela 3). Essas diferenças podem estar associadas ao menor tempo de alimentação das lagartas nas cultivares, bem como a presença de fatores que conferem resistência e/ou suscetibilidade. A sobrevivência, também foi maior em virtude da menor migração das lagartas durante o período que antecede à pupação, concordando com 
Williams et al.(1989), que recomendaram a utilização deste parâmetro antes dos 14 dias após a infestação. $\mathrm{O}$ peso das lagartas foi considerado pelos mesmos autores, como o parâmetro mais eficiente nos estudos de resistência de milho a $S$. frugiperda do que a sobrevivência, de vez que esta sofre influência de fatores bióticos, como por exemplo a predação. As cultivares CMS-22 e CMS-35 provavelmente, também devem apresentar mecanismos de resistência do tipo antibiose, pelo fato de apresentarem os menores pesos de lagartas em comparação a Dina-70 e AG-510.

$\mathrm{O}$ peso e o comprimento das lagartas também foram os parâmetros que apresentaram uma maior correlação $(\mathrm{r}=0,7776, \quad \mathrm{P}=0,01)$ (Tabela 2).

\section{RESUMO}

Dois experimentos foram estudados em Casa-deVegetação, com o objetivo de identificar fontes de resistência em 15 cultivares de milho à Spodoptera frugiperda. Utilizaram-se as cultivares CMS-35, CMS-36, Dina-70, Cargill525, Pioneer-3210, Pioneer-6875, BR-201, BR-
451, AG-106, AG-510, CMS-22, Contimax-322, Jatinã $C_{3}$ Anão, Cargill-511 e BR-106. As plantas foram infestadas com 30 lagartas recém eclodidas por planta, quando estas apresentavam de 6 a 8 folhas. Após 10 e 14 dias da infestação, foram avaliados os danos através de uma escala visual de notas de 0 a 9 . Não houve diferença estatística significativa entre as cultivares para os parâmetros estudados: dano, peso, comprimento e sobrevivência das lagartas. No entanto, as correlações lineares entre o peso $\mathrm{x}$ comprimento de lagartas, foram significativas $(\mathrm{n}=0,7776, \mathrm{P}=$ 0,01 ), demonstrando que esses parâmetros podem ser utilizados em estudos de resistência de milho à Spodoptera frugiperda.

\section{AGRADECIMENTOS}

À Universidade Federal Rural de Pernambuco pela colaboração na condução dos experimentos. À Empresa de Pesquisa Agropecuária do Estado de Alagoas (EPEAL) pelo incentivo financeiro e à Empresa Pernambucana de Pesquisa Agropecuária (IPA) pelo fornecimento das cultivares e apoio técnico. Aos estagiários Édney Vila Nova Cavalcanti e Ricardo Souza Cavalcanti do IPA, pelas colaborações de digitação.

Tabela 3. Médias dos danos (nota), sobrevivência (\%), peso (mg) e comprimento de lagartas (mm) de Spodoptera frugiperda em 15 cultivares de milho, 10 dias após infestação artificial, em casa-de-vegetação. Recife, PE, de setembro a outubro de 1993

\begin{tabular}{lcccc}
\hline \multicolumn{1}{c}{ Cultivar } & Dano & Sobrevivência $^{*}$ & Peso & Comprimento \\
\hline CMS-35 & 6,12 & 7,85 & 43,25 & 16,52 \\
CMS-36 & 7,50 & 8,28 & 77,75 & 16,45 \\
Dina-70 & 6,37 & 5,38 & 99,00 & 18,18 \\
Cargill-525 & 7,12 & 6,62 & 55,50 & 14,80 \\
Pioneer 3210 & 7,87 & 5,40 & 62,25 & 16,40 \\
Pioneer 6875 & 7,37 & 7,05 & 54,25 & 15,72 \\
BR-201 & 6,25 & 6,63 & 57,75 & 16,07 \\
Br-451 & 6,87 & 5,78 & 69,75 & 13,87 \\
AG-106 & 6,37 & 6,63 & 45,50 & 16,57 \\
AG-510 & 7,25 & 9,10 & 80,25 & 11,47 \\
CMS-22 & 6,62 & 7,88 & 28,50 & 16,30 \\
Contimax-322 & 8,00 & 4,95 & 64,00 & 16,02 \\
JatinãC Anão $_{\text {Cargil 511 }}^{6,50}$ & 5,87 & 4,95 & 72,75 & 15,40 \\
BR-106 & 7,25 & 6,65 & 47,00 & \\
& & 6,63 & 54,00 & NS \\
\hline \multicolumn{1}{c}{ F } & NS & & & 19,58 \\
\hline \multicolumn{1}{c}{ C.V.(\%) } & 15,62 & NS & 40,97 & NS \\
\hline
\end{tabular}

* Dados transformados em Arc sen $\sqrt{\frac{p}{100}}$ 


\section{REFERÊNCIAS}

Cruz, I. (1993), Controle econômico da lagarta do cartucho. Correio Agrícola, 1, 8-11.

Cruz, I \& Alvarenga, C.D. (1990) Avaliação da variabilidade genética da população de milho doce BR 400 para resistência à lagarta-docartucho,Spodoptera frugiperda (J.E. Smith, 1797). In: $18^{0}$ Congresso Nacional de Milho e Sorgo, 87, Vitória.

Cruz, I. \& Turpin, F.T. (1982), Efeito da Spodoptera frugiperda em diferentes estágios de crescimento da cultura do milho. Pesq. agropec. bras., 17, 355-359.

Davis, F. M. \& Williams, W. F. (1989), Methods used to screen maize for resistance and to determine mechanisms of resistance to the Southwestern corn boerer and fall armyworm.In:International Symposium on Methodologies for Development Host Plant Resistance to Maize Insects, México. Toward insect resistance maize for the third world.101-104, México, CIMMYT.

Melo, M. \& Silva, R.F.P. da (1987), Influência de três cultivares de milho no desenvolvimento de Spodoptera frugiperda (J. E. Smith, 1797) (Lepidoptera: Noctuidae). An. Soc. Entomol. Brasil, 16, 37-49.

Mihm, J.A. (1983), Efficient mass rearing and infestation techniques to screen for resistence to fall armyworm, Spodoptera frugiperda. México. Centro Internacional de Mejoramiento de Maiz y Trigo, P 16.

Mihm, J.A. (1984), Técnicas eficientes para la crianza masiva, e infestación de insectos, en la selección de las plantas hospedantes para resistencia al gusano cogollero Spodoptera frugiperda. México: CIMMYT, P 16.

Morril, W. L. \& Greene, G. L. (1974), Survival of fall armyworm larvae and yields of field corn after artificial infestation. J. Econ. Entomol., 67, 119-123.

NG, S.S \& Davis, F.M; Williams, W.P. (1985), Survival, growth, and reproduction of the fall armyworm (Lepidoptera: Noctuidae) as affected by resistant corn genotypes. J. Econ. Entomol., 78, 967-971.

Silva, W. J.; Reis, P. R. \& Rossetto, C. J.(1969), Resistência de milho a Spodoptera frugiperda (J.E. Smith). I Tehva Barbados 3D provável fonte de resistência. In: $2^{\mathrm{a}}$ Reunião da
Sociedade Brasileira de Entomologia, 22, Recife,PE.

Viana, P.A. \& Potenza, M. R. (1991), Identificação de fontes de resistência de milho a Spodoptera frugiperda (Lepidoptera: Noctuidae).In: $13^{0}$ Congresso Brasileiro de Entomologia, 530. Recife, PE.

Videla, G.W.; Davis, F. M.; Williams, W.P \& NG, S.S. (1992), Fall armyworm (Lepidoptera: Noctuidae) larval growth and survivorship on susceptible and resistant corn at different vegetative growth stages. J. Econ. Entomol., 85, 2486-2491.

Widstrom, N.W.; Wiseman, B.R. \& Mc. Millian, W.W. (1972), Resistance among some maize inbreds and single crosses to fall armyworm injury. Crop. Sci., 12, 290-293.

Williams, W. P.; Buckley, P. M. \& Davis, F. M. (1989), Combinig ability for resistance in corn to fall armyworm and southwestern corn borer. Crop. Sci., 29, 913-915.

Williams, W.P; Davis, F.M. \& Scott, G.E. (1978), Resistance of corn to leaf-feeding damage by the fall armyworm. Crop. Sci., 18, 861-863.

Wilson, R. L.; Wiseman, B.R. \& Reed, G. L. (1991), Evaluation of J.C Eldredge popcorn collection for resistance to corn earworm, fall armyworm (Lepidoptera: Noctuidae), and european corn borer (Lepidoptera: Pyralidae). J. Econ. Entomol., 84, 693-698.

Wiseman, B.R. (1985), Development of resistence in corn and sorghum to foliar - and ear / panicle-feeding worm complex. In: $40^{\text {th }}$ Annual Corn and Sorghum Reseach Conference, 108-124, Chicago.

Wiseman, B.R. \& Isenhour, D.J. (1988), Feeding responses of fall armyworm larvae on excised green and yellow whrol tissue of resistant and susceptible corn. Fla. Entomol., 71, 243-246.

Wiseman, B. R; Painter, R. H. \& Wasson, C. E. (1966), Detecting corn seedling differences in the greenhouse by visual classification of damage by the fall armyworm. J. Econ. Entomol., 59, 1211-1214.

Wiseman, B.R; Wasson, C.E \& Painter, R.H.(1967), An unusuval feeding habit to measure differences in damage to 81 LatinAmerican lines of corn by the fall armyworm, Spodoptera frugiperda (J.E Smith). Agron. J., 59, 279-281. 
Wiseman, B. R. \& Widstrom, N. W. (1986), Mechanisms of resistance in "Zapalote Chico" corn silks to fall armyworm (Lepidoptera: Noctuidae) larvae. J. Econ. Entomol.,79, 1390-1393.

Wiseman, B.R.; Williams, W. S. \& Davis, F. M. (1981), Fall armyworm: resistance mechanisms in selected corns. J. Econ. Entomol., 74, 622-624.
Yang, G.; Isenhour, J. \& Spelie, K. E. (1991), Activity of maize cuticular lipids in resistance to leaf-feeding by the fall armyworm. Fla. Entomol.,74, 229-236.
Received: December 23, 1997; Revised: April 06, 1998; Accepted: December 02, 1998. 